\title{
Aprendizagem baseada em equipes no ensino de bioquímica na graduação
}

\author{
Team-based learning in biochemistry teaching at undergraduate level
}

\author{
Evelyn Winter ${ }^{1 *}$, Frank Paulo Cardoso ${ }^{2}$
}

${ }^{1}$ Docente do Departamento de Agricultura, Biodiversidade e Florestas, Centro de Ciências Rurais, Universidade Federal de Santa Catarina, Campus de Curitibanos, Curitibanos, SC, Brasil.

${ }^{2}$ Monitor da disciplina de bioquímica, Centro de Ciências Rurais, Universidade Federal de Santa Catarina, Campus de Curitibanos, Curitibanos, SC, Brasil.

*e-mail: evelyn.winter@ufsc.br

\section{Resumo}

O ensino da bioquímica é, muitas vezes, exposto em aulas teóricas, sendo visto pelos alunos como disciplina de difícil aprendizado. A aprendizagem baseada em equipe (ABE) é uma ferramenta de aprendizagem ativa que combina a preparação independente do estudante, previamente à aula, com a discussão em pequenos grupos em classe, sendo que o estudante assume uma posição ativa no aprendizado. Adotamos a $A B E$ no ensino do metabolismo lipídico na disciplina de bioquímica. Neste estudo, avaliamos as percepções dos alunos sobre a $A B E$ através de uma pesquisa qualitativa e também através do desempenho estudantil em três semestres onde diferentes abordagens de ensino foram utilizadas. Para a preparação da atividade, aulas expositivas foram complementadas com materiais extras que foram disponibilizados previamente aos estudantes. No dia da atividade os estudantes realizaram um teste individual, um teste de equipe e finalmente uma discussão geral do assunto abordado. Os resultados demonstram que os estudantes se sentiram motivados, no entanto apresentaram dificuldades em trabalhar em grupo. O desempenho dos estudantes que participaram da $A B E$ melhorou em relação aos alunos que participaram apenas de aulas expositivas ou estudo dirigido. Este trabalho permite concluir que a inserção de metodologia ativa como a ABE favoreceu o desenvolvimento de habilidades cognitivas e não cognitivas e pode ser uma estratégia de ensino eficaz para a bioquímica.

Palavras-chave: (Aprendizagem baseada em equipes; bioquímica; metodologias ativas)

\begin{abstract}
The teaching of biochemistry is often exposed in theoretical classes, being seen by the students as a discipline of difficult learning. The team-based learning (TBL) is an active learning tool that combines the independent preparation of the student before the class with the discussion in small groups in class, and the student assumes an active position in the learning. We adopted the TBL in the teaching of lipid metabolism in the discipline of biochemistry. In this study, we evaluated the students' perceptions about TBL through a survey and also through a comparison of the student performance of three semester students who answered the same questions within a test. For the preparation of the activity, lectures were given and supplemented with reading of textbooks and videotapes. On the day of the activity, the students performed the individual test, the team test and finally a general discussion of the subject addressed. The results demonstrate that the students felt motivated; however, they presented difficulties in working in group. The students' performance in the test improved in relation to students who participated only in lectures or complemented with a directed study. This work allows concluding that the insertion of active methodology like TBL favors the development of cognitive and non-cognitive abilities and can be an effective teaching strategy for biochemistry.
\end{abstract}

Keywords: (Team-based learning; biochemistry; active methodology) 


\section{Ficha da atividade}

\begin{tabular}{|l|l|}
\hline Título & Aprendizagem baseada em equipes no ensino de bioquímica na graduação. \\
\hline Categoria: & Prática de Ensino \\
\hline Tipo & Atividades didáticas inovadoras: métodos diferenciados \\
\hline Público-alvo: & Estudantes de graduação na disciplina de bioquímica. \\
\hline $\begin{array}{l}\text { Conteúdos } \\
\text { abordados }\end{array}$ & Metabolismo de Lipídios \\
\hline $\begin{array}{l}\text { Objetivos } \\
\text { educacionais }\end{array}$ & Revisar e consolidar o conhecimento dos conteúdos de metabolismo de lipídios. \\
\hline Duração & 60 minutos \\
\hline $\begin{array}{l}\text { Materiais } \\
\text { utilizados }\end{array}$ & Questionário e fichas de apostas impressas \\
\hline Criação & $01 / 07 / 2018$ \\
\hline Aplicação & $20 / 08 / 2018$ e 29/10/2018 \\
\hline Link & \\
\hline
\end{tabular}




\section{Introdução}

Os modelos de ensino tradicionais tratam o conhecimento como um conjunto de informações que são transmitidas pelos professores aos estudantes. Estes modelos colocam o aluno como uma figura passiva sendo o professor, o grande protagonista da aula. Nas metodologias ativas de ensino o aluno tem autonomia e passa a ser o construtor do seu próprio conhecimento, ampliando o papel do professor que passa a ter um papel de tutor [1]. Este tipo de metodologia tem sido aplicada principalmente na área da saúde e começou a ser utilizada em 1969 na Universidade de McMaster no Canadá. No Brasil ela é usado desde 1997, sendo a Universidade de Marília uma das pioneiras . As metodologias ativas de aprendizagem incluem diversas abordagens mas as principais são as que trabalham com resolução de problemas, aprendizagem baseada em projetos e aprendizagem baseada em equipes ( $\mathrm{ABE}$ ) - do inglês, team based learning (TBL) -, que como o próprio nome revela, se trata da formação de equipes dentro de determinada turma para que o aprendizado seja feito em conjunto e haja compartilhamento de ideias $[1,3]$.

A ABE é um método ativo que foi inicialmente implementado por Larry Michaelsen, sendo uma atividade que pode ser usada desde turmas pequenas de 25 alunos até turmas grandes de 100 alunos [4]. Em 2001 o governo dos Estados Unidos financiou professores envolvidos em ciências da saúde para que introduzissem novas formas de ensino e com isso a $A B E$ foi contemplada [3]. Este método tem um alto potencial de aprendizagem pois faz com que o aluno pesquise e discuta seus conhecimentos com os colegas de grupo, permitindo uma fixação do conteúdo ampliada. Um dos principais desafios encontrados na $\mathrm{ABE}$, assim como nas metodologias ativas, é atingir a autonomia do estudante e a mudança do papel do professor que passa a ser o facilitador de todo este processo de ensino-aprendizagem. A execução desta atividade se torna possível quando se segue as 4 regras essenciais da $A B E:$ i) a formação dos grupos deve ser adequada e os mesmos devem ser bem administrados; ii) os alunos devem ser responsáveis pelo desempenho individual e da equipe; iii) os estudantes devem receber um feedback do professor; iv) a equipe deve interagir para que se promova o aprendizado não somente individual, mas principalmente do grupo [5].

A aprendizagem de bioquímica é definida pelos estudantes como algo complexo, sendo ela uma disciplina básica em diversos cursos das ciências da vida e de grande importância para a formação e vida profissional. Em geral, as aulas são centradas no professor através de aulas expositivas fazendo do aluno um elemento passivo [6]. 
Algumas metodologias ativas já vem sendo usados no ensino de Bioquímica como a aprendizagem baseada em problemas[7] e a ABE que apresentou um efeito positivo em turmas de bioquímica na Chicago College of Osteopathic Medicine e Midwestern University nos Estados Unidos. Winkle e colaboradores relataram que estudantes de medicina que participaram da $A B E$ aprenderam mais por este método e refletiram mais no paciente [8]. Sendo assim, o objetivo deste trabalho foi avaliar a inserção da ABE na disciplina de bioquímica ministrada para o curso de medicina veterinária da Universidade Federal de Santa Catarina.

\section{Desenvolvimento}

O material para a aplicação da atividade foi preparado abordando conteúdos relacionados ao metabolismo de lipídios. Os alunos $(n=60)$ assistiram a 2 aulas expositivas sobre o tema e foram orientados a complementar o assunto, estudando capítulos do livro texto e assistindo videoaulas referentes ao conteúdo. Para a atividade $\mathrm{ABE}$, elaborou-se um questionário com 11 perguntas objetivas contendo 5 alternativas de múltipla escolha.

Os cartões de apostas foram elaborados em editor de texto de forma a otimizar a impressão em uma folha A4.

Todos os materiais foram impressos pelo professor e distribuídos para os estudantes na aplicação da atividade conforme orientações descritas abaixo.

\section{Procedimento de uso do material ou aplicação da atividade}

A aplicação desta metodologia obedece alguns passos para que a atividade tenha êxito conforme demonstrado na figura 1. São quatro os elementos essenciais para a utilização da ABE: (i) atividades da fase de preparação; (ii) teste de preparação individual (TPI); (iii) teste de preparação em equipes (TPE); e (iv) avaliação final da atividade. Além das fases descritas acima, a $A B E$ ainda pode ter uma última fase realizada extraclasse onde os estudantes podem fazer a resolução de casos e/ou exercícios e tem como finalidade a fixação dos conteúdos estudados (Figura 1). 


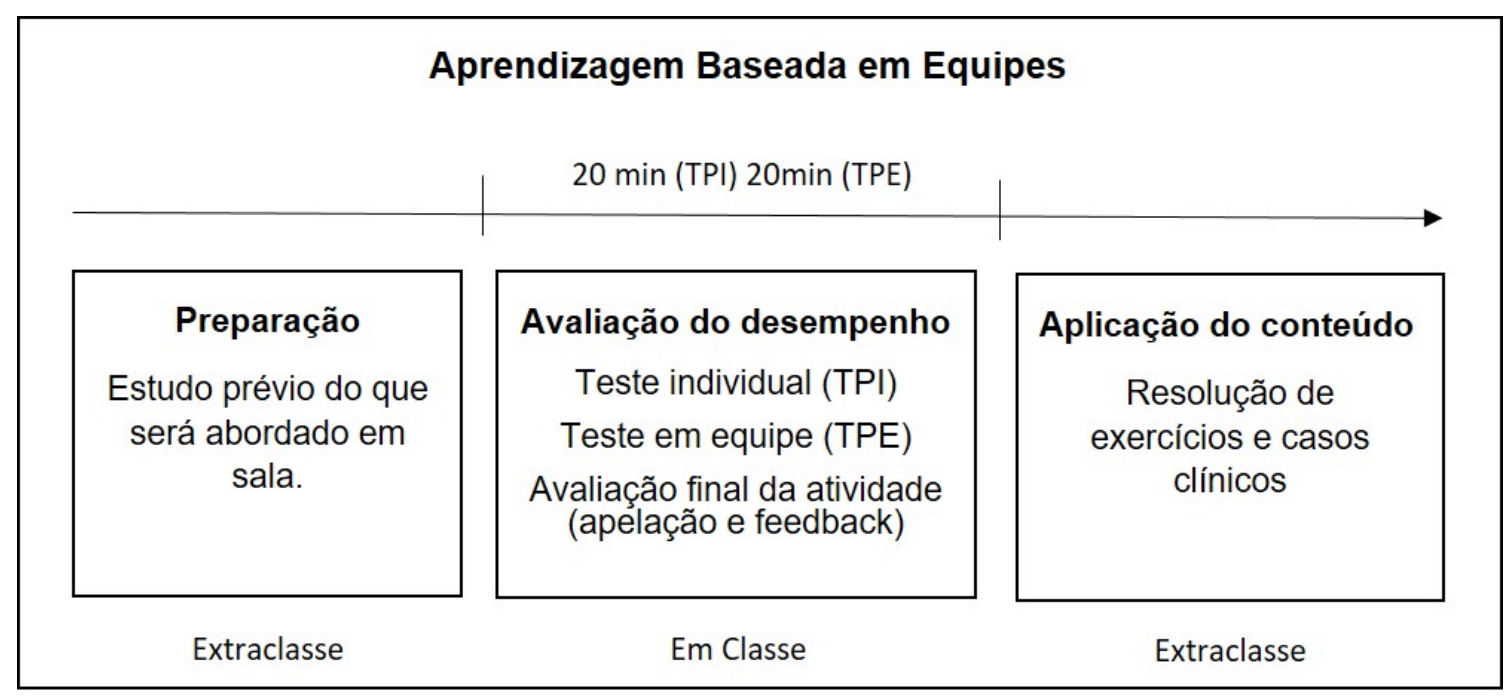

Figura 1. Etapas de execução da Aprendizagem Baseada em Equipes.

$\mathrm{Na}$ fase de preparação os estudantes realizaram um estudo prévio extraclasse do conteúdo que seria abordado em aula. Os materiais para estudo podem ser textos, vídeos entre outros e devem ser entregues ao aluno com no mínimo dois dias de antecedência. Para esta atividade os alunos receberam com uma semana de antecedência textos e vídeoaulas para estudo.

As próximas etapas da atividade aconteceram em classe e deram sequência a fase de preparação. Inicialmente os alunos responderam a um teste caracterizando o teste de preparação individual (TPI). Os alunos receberam um tempo de 20 minutos para responder individualmente questões objetivas referentes ao tema proposto. As questões possuíam 5 alternativas de múltipla escolha, sendo que mais de uma alternativa poderia estar correta. Nesta fase os alunos receberam uma ficha de apostas onde eles puderam escolher uma ou mais alternativas certas para apostar seus pontos (Figura 2).

\begin{tabular}{l} 
Nome: Aprendizagem baseada em equipes - Cartão de apostas \\
Cada questão vale 5 pontos e pode ter mais de 1 alternativa correta. Você pode apostar esses 5 pontos em \\
quantas alternativas desejar. \\
\hline \\
\hline
\end{tabular}

Figura 2. Cartão de apostas. 
A etapa seguinte consistiu em formar equipes aleatórias, escolhidas pelo professor, de 5 a 6 estudantes que em conjunto, responderam ao mesmo teste, caracterizando o teste de preparação em equipe (TPE). Nessa fase os alunos dialogaram com os colegas de equipe e receberam uma nova ficha de apostas que foi preenchida com o consenso de todos. O tempo para essa etapa foi de 20 minutos e os alunos foram orientados a realizar a marcação das apostas apenas com caneta esferográfica e sem rasuras.

A última fase da atividade consistiu na correção e discussão dos conteúdos. Cada equipe corrigiu o gabarito de outra equipe e verificaram a pontuação final obtida. As equipes que atingiram pontuação máxima, obtiveram nota 10 e a mesma proporção foi usada para calcular todas as notas. Caso os alunos tivessem alguma objeção, é possível, nesta etapa, fazer uma apelação e discussão da situação. Ao final, os alunos entregaram os cartões de apostas do TPE para o professor registrar a nota. A última fase da atividade terminou com o professor elucidando conteúdos que apresentaram maior dificuldade e fazendo uma avaliação final da atividade. Para que o processo apresente resultados positivos, em termos de aprendizagem e trabalho colaborativo, é importante que o professor gerencie a formação das equipes heterogêneas e conduza os testes individuais, em equipe e a última etapa de feedback e apelação.

\section{Procedimentos de avaliação}

A avaliação desta atividade aconteceu de duas maneiras: i) realização de um questionário estruturado que avaliou a opinião dos alunos em relação a sua experiência de participação na $A B E ;$ ii) análise do desempenho dos estudantes em prova.

O questionário contou com cinco perguntas referentes as impressões que os estudantes obtiveram após participarem da $A B E$, e uma pergunta aberta onde poderiam detalhar suas percepções. O questionário foi enviado online para os estudantes responderem de forma voluntária e anônima. Os estudantes poderiam escolher até duas alternativas em cada pergunta do questionário.

A análise do desempenho dos alunos foi realizada comparando-se a porcentagem de acertos em uma questão objetiva e uma questão discursiva as quais foram apresentadas aos alunos em três diferentes semestres. A primeira turma teve acesso ao conteúdo apenas através de aulas expositivas; a segunda turma também assistiu às aulas expositivas, mas adicionalmente recebeu um estudo dirigido para ser respondido em casa; a terceira turma igualmente assistiu às aulas expositivas e participou da atividade em equipe discutida neste trabalho ( $A B E)$. 


\section{Potencial educacional}

A bioquímica é um modelo de disciplina do ciclo básico ofertada em quase todos os cursos das ciências da vida e em consequência da grande importância do seu estudo e à grande complexidade de compreensão e desinteresse pela disciplina por parte dos alunos, diferentes métodos têm sido propostos para melhorar o ensino dessa disciplina. Poucos trabalhos relataram especificamente o benefício da ABE aplicada ao ensino de bioquímica $[9,10]$, no entanto, muitos trabalhos demonstraram que as metodologias ativas de aprendizagem envolvem mais os estudantes e tem resultados promissores [11-14].

\section{Diferencial com as atividades/materiais preexistentes}

$A$ ABE se enquadra dentro das metodologias ativas de aprendizagem e se diferencia das metodologias convencionais pois proporciona maior autonomia do estudante. Além disso, a atividade demanda um alto nível de comprometimento individual para o bom rendimento do grupo, a confiança entre os membros, o trabalho em equipe e o desenvolvimento da comunicação [15]. Em alguns momentos, os estudantes citaram que tiveram um desempenho pior em equipe pois tiveram dificuldade em expor sua opinião, demonstrando quão importante é o estímulo ao debate, a comunicação entre os pares e a resolução de conflitos.

A montagem de equipes heterogêneas fortalece a troca de conhecimentos entre alunos com mais lacunas e aqueles com mais facilidade [16]. Para evitar a insatisfação dos alunos quanto a decisão arbitrária do professor ao montar as equipes, é importante explicar os benefícios da escolha, mostrando que independente da profissão e/ou local de trabalho, nem sempre haverá opção de escolher os colegas.

A aprendizagem baseada em equipes já é uma ferramenta utilizada em diversas disciplinas [15,17-19]. Normalmente esta atividade é realizada com o uso de cartões de correção instantânea, que funcionam como se fossem "raspadinhas". Cada questão é respondida ao mesmo tempo por todas as equipes, que "raspam" a alternativa que considerarem certa. Se os alunos rasparem e não encontrarem a resposta certa, voltam a discutir a questão [15,19]. Apesar de ser uma metodologia atrativa e dinâmica, a execução da $A B E$ convencional demanda tempo e materiais para a confecção dos cartões. Além disso, algumas equipes demoram mais tempo para discutir as questões que outras gerando uma dispersão dos estudantes.

Propomos uma atividade simplificada, que não demanda um grande preparo das fichas de apostas, na qual cada equipe gerencia o seu tempo para cada resposta. 


\section{Resultados da avaliação}

Vinte e sete estudantes aceitaram responder ao questionário online. Como pode ser observado na Figura 3C, a dificuldade mais relatada pelos alunos foi a pouca interação com a equipe (43,5\%). Essa dificuldade de trabalho em equipe também fica evidente na Figura 3B onde os alunos demonstram que preferiam que as equipes fossem escolhidas por afinidade $(57,1 \%)$ e na Figura $3 A$ onde percebemos que apenas $24 \%$ dos alunos gostam de trabalhar em grupo. Apesar do exposto, alguns relatos deixam claro a importância do trabalho em equipe: "Gostei bastante, achei que foi uma forma diferente de aprendizagem, a discussão com os colegas ajudava a entender melhor o conteúdo de uma maneira mais simples", "Além de ajudar a memorizar o conteúdo, já podemos debater entre os colegas e analisar nossos erros". Um dos elementos essenciais para se atingir os objetivos da $A B E$ é a divisão estratégica das equipes realizada pelo instrutor, garantindo a aprendizagem cooperativa [16].

Em relação ao aprendizado, os alunos relataram que a atividade estimulou o aprendizado (76\%) e a memorização dos conteúdos (44\%): "Verifiquei pontos em qual tinha dificuldade e não sabia".

Em relação ao envolvimento dos alunos na atividade, $24 \%$ dos que responderam o questionário indicaram que gostam de se sentir mais inseridos na aula. Alguns trabalhos confirmam que o estudante se sente motivado com o uso de metodologias ativas de aprendizagem, inclusive quando a mesma é aplicada ao ensino de bioquímica [9, 15]. Neste trabalho alguns relatos deixam evidente essa realidade: "Achei interessante $e$ participaria mais vezes. É tão cativante pelo fato de incentivar a aprendizagem de outra forma", "Foi uma atividade descontraída e possibilitou a discussão do conteúdo e compartilhamento de conhecimento entre os integrantes". Ao final do questionário, 100\% dos alunos responderam que participariam novamente de atividades como esta. Percepções positivas dos estudantes também foram relatados em outros trabalhos que avaliaram atividades de ABE [17].

As atividades de ABE podem ser previstas para acontecer em diferentes tempos, normalmente entre 45-75 minutos [15, 16]. Uma das principais dificuldades relatadas pelos estudantes neste trabalho foi a inadequação do tempo à atividade. $34,8 \%$ consideraram pouco tempo para a execução da atividade proposta. O questionário foi elaborado considerando 1 hora para a execução da atividade, no entanto esse tempo pode ser modificado de acordo com a dificuldade da atividade. Percebe-se, no entanto, que $34,8 \%$ dos alunos também relataram como dificuldade o fato de não terem estudado 
o tema previamente (Figura 4). A dificuldade com o tempo, pode estar, portanto, relacionada a uma falha na fase de preparação individual que acontece antecipadamente à $A B E$.

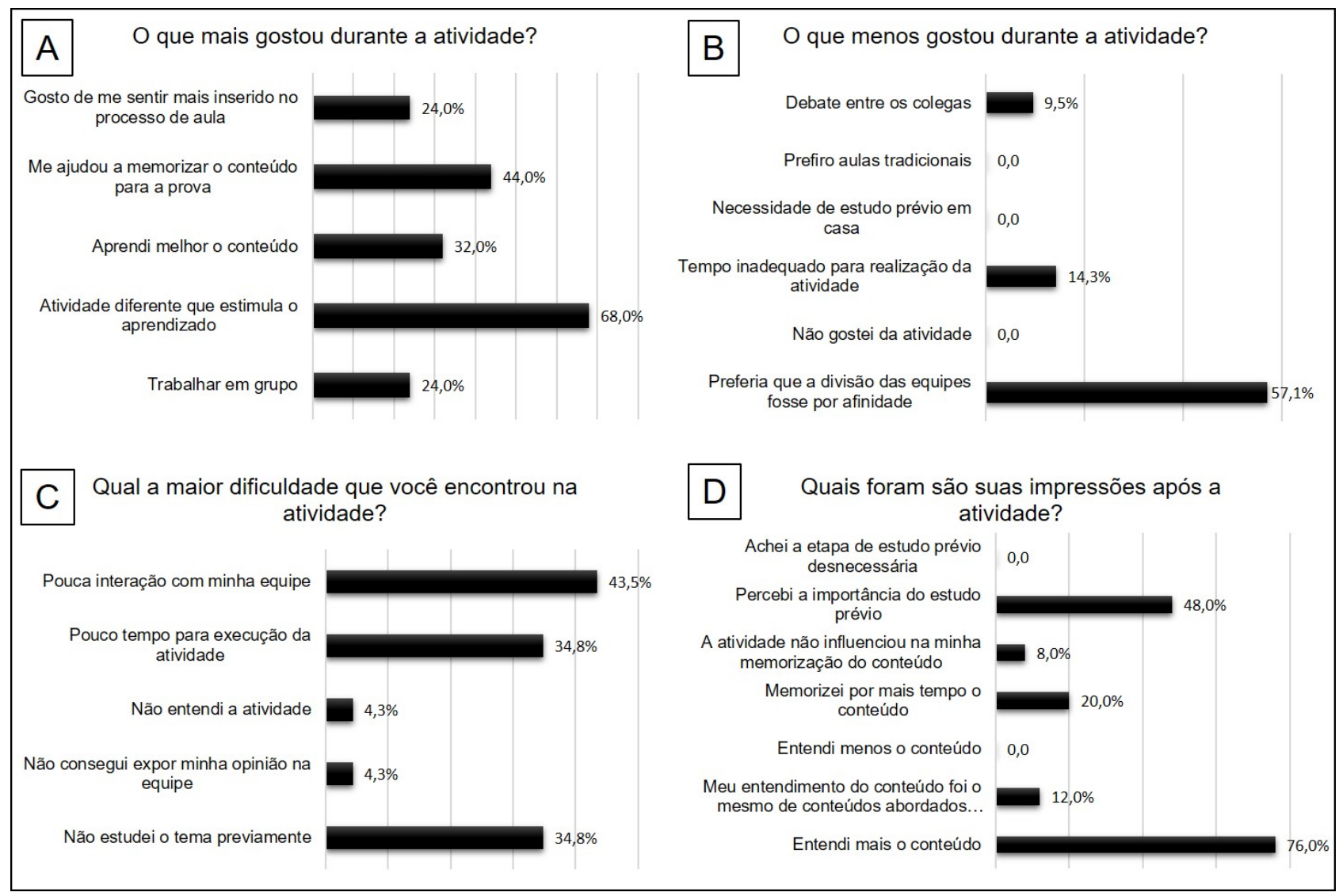

Figura 3. Avaliação da atividade Aprendizagem Baseada em Equipes, aplicada na disciplina de Bioquímica, por estudantes de graduação.

A segunda forma de avaliação da atividade foi a análise do desempenho dos estudantes em duas questões relacionadas ao tema abordado. 19,6\% dos estudantes acertaram a questão objetiva quando assistiram apenas a aula expositiva. Esse valor aumentou para 40,0 e 69,4\% quando as aulas expositivas foram complementadas com estudo dirigido e $A B E$, respectivamente.

O mesmo perfil de resultado se observa no rendimento dos alunos em relação a questão discursiva (Figura 4). Percebe-se que o desempenho dos estudantes foi maior quando aconteceu a aprendizagem baseada em equipes. Resultados semelhantes foram observados em outros trabalhos que avaliaram o desempenho dos estudantes em atividades de ABE e outras atividades envolvendo metodologias de ensino tradicionais $[20,22]$. 


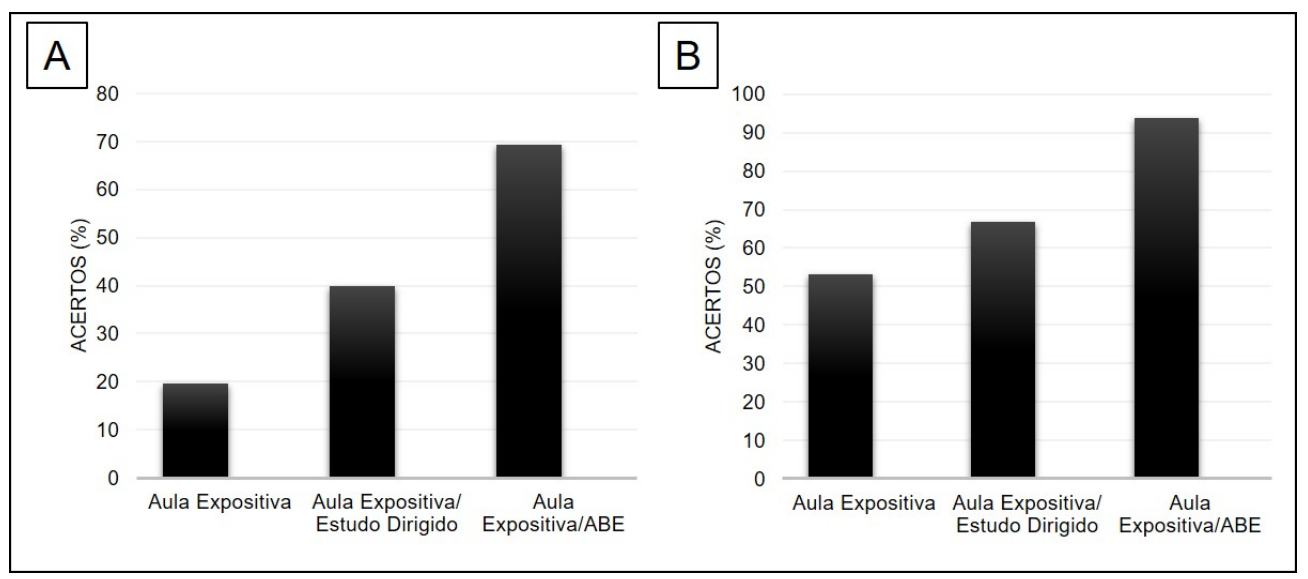

Figura 4. Desempenho dos estudantes em duas questões de bioquímica com o uso de diferentes metodologias de ensino. A) Questão objetiva; B) Questão discursiva.

\section{Considerações finais}

As observações obtidas neste trabalho permitem concluir que a aprendizagem baseada em equipes influenciou positivamente o aprendizado e a motivação de estudantes de bioquímica do curso de medicina veterinária.

\section{Referências}

[1] Freitas CM, Freitas CASL, Parente JRF, Vasconcelos MIO, Lima GK, Mesquita KOd, et al. USO DE METODOLOGIAS ATIVAS DE APRENDIZAGEM PARA A EDUCAÇÃO NA SAÚDE: ANÁLISE DA PRODUÇÃO CIENTÍFICA. Trabalho, Educação e Saúde. 2015;13:117-30.

[2] Melo BC SAG. A prática da Metodologia Ativa: compreensão dos discentes enquanto autores do processo ensinoaprendizagem. Com Ciências Saúde. 2012;23(4):327-39.

[3] BOLLELA VRS, M.H.; TOURINHO, F.S.V.; AMARAL, E. Aprendizagem baseada em equipes: da teoria à prática. Medicina (Ribeirão Preto). 2014;47(3):293-300.

[4] Michaelsen LK, Watson WE, Black RH. A realistic test of individual versus group consensus decision making. Journal of Applied Psychology. 1989;74(5):834-9.

[5] Michaelsen LK, Sweet M. The essential elements of team-based learning. New Directions for Teaching and Learning. 2008;2008(116):7-27.

[6] de Andrade RSB, e Silva AFdS, Zierer MdS. Avaliação das dificuldades de aprendizado em Bioquímica dos discentes da Universidade Federal do Piauí. Revista de Ensino de Bioquímica; v 15, n 1 (2017): REB (Jan - Jun)DO - 1016923/rebv15i1690. 2017.

[7] Silveira Covizzi UD, Lopes de Andrade PdF. Estratégia para o ensino do metabolismo dos carboidratos para o curso de farmácia, utilizando metodologia ativa de ensino. Revista de Ensino de Bioquímica; v 10, n 1 (2012): RBEBBM (Cessou em 2012 Cont ISSN 2318-8790 Revista de Ensino de Bioquímica)DO 1016923/rebv10i1169. 2012.

[8] Van Winkle LJ, Chandar N, Green JM, Lynch SM, Viselli SM, Burdick P. Does Critical Reflection by Biochemistry Learning Teams Foster Patient-centered Beliefs among Medical Students? Medical Science Educator. 2011;21(2):158-68.

[9] Chhabra N, Kukreja S, Chhabra S, Chhabra S, Khodabux S, Sabane H. Team-based Learning Strategy in Biochemistry: Perceptions and Attitudes of Faculty and 1(st)-Year Medical Students. International journal of applied \& basic medical research. 2017;7(Suppl 1):S72-S7. 
[10] Mun KH, Mun KC. Verification of learner's differences by team-based learning in biochemistry classes. Korean J Med Educ. 2017;29(4):263-9.

[11] Matthews JC. Intermeshing Passive and Active Learning Strategies in Teaching Biochemistry. American Journal of Pharmaceutical Education 1997;61.

[12] Bobich JA. Active Learning of Biochemistry Made Easy (for the Teacher). Journal of Chemical Education. 2008;85(2):234.

[13] Uderlei Donizete Silveira Covizzi PdFLdA. Estratégia Para O Ensino Do Metabolismo Dos Carboidratos Para O Curso De Farmácia, Utilizando Metodologia Ativa De Ensino. REvista Brasileira de Ensino de Bioquímica e Biologia Molecular. 2012;1.

[14] Garcês BP, Santos KdO, Oliveira CAd. Aprendizagem baseada em projetos no ensino de bioquímica metabólica. Revista Ibero-Americana de Estudos em Educação; v13, n esp 1, maio (2018). 2018.

[15] Mclnerney MJ, Fink LD. Team-based learning enhances long-term retention and critical thinking in an undergraduate microbial physiology course. Microbiology education. 2003;4:3-12.

[16] Michaelsen LK, Sweet M. Team-based learning. New Directions for Teaching and Learning. 2011;2011(128):41-51.

[17] Vasan NS, DeFouw DO, Compton S. A survey of student perceptions of team-based learning in anatomy curriculum: Favorable views unrelated to grades. Anatomical Sciences Education. 2009;2(4):150-5.

[18] Zgheib NK, Simaan JA, Sabra R. Using team-based learning to teach pharmacology to second year medical students improves student performance. Medical Teacher. 2010;32(2):130-5.

[19] OLIVEIRA TEA, I.S. Aprendizagem Baseada em Equipes (Team-Based Learning) : um método ativo para o Ensino de Física. Caderno Brasileiro de Ensino de Física,. 2016;33(3).

[20] Vasan NS, DeFouw DO, Compton S. Team-based learning in anatomy: An efficient, effective, and economical strategy. Anatomical Sciences Education. 2011;4(6):333-9.

[21] Faezi ST, Moradi K, Ghafar Rahimi Amin A, Akhlaghi M, Keshmiri F. The effects of team-based learning on learning outcomes in a course of rheumatology. Journal of advances in medical education \& professionalism. 2018;6(1):22-30.

[22] Koles PG, Stolfi A, Borges NJ, Nelson S, Parmelee DX. The Impact of Team-Based Learning on Medical Students' Academic Performance. Academic Medicine. 2010;85(11):1739-45.

\section{Agradecimentos}

Agradeço aos estudantes pelo seu entusiasmo e disposição em participar das atividades e responder aos questionários de avaliação.

\section{Materiais suplementares}

\begin{tabular}{|c|c|c|}
\hline Material & Descrição & Tipo \\
\hline 2 & Questionário - Metabolismo de lipídios & Questionário \\
\hline 3 & Cartão de apostas & Cartão de apostas \\
\hline
\end{tabular}

